\title{
Jahresbericht über die Aktivitäten der FMH im Bereich MPA/MPK
}

\author{
Aisha Ahmed \\ Dr. med., Präsidentin der kantonalen Delegierten für MPA-Fragen
}

\begin{abstract}
Immer noch prägt die COVID-19-Pandemie unseren Alltag. Es ist für alle eine Herausforderung, stets auf dem aktuellen Stand der Massnahmen, Einschränkungen und Vorgaben zu bleiben. Erfreulicherweise sind durch diese Krise auch neue Ideen gefördert worden und lange fällige Verbesserungen erfolgt. Wir haben die Vor- und Nachteile der digitalen Kommunikation erlebt, viele haben ihre Arbeitsweise umgestellt und neue Prioritäten gesetzt.
\end{abstract}

Wie Sie sehen werden, hat sich die FMH weiterhin intensiv für die Belange der MPA und MPK eingesetzt. Gerne stelle ich das Personelle an den Anfang dieses Berichts: Der Zentralvorstand der FMH hat dem Vorschlag der Versammlung der Delegierten für MPA-Fragen der kantonalen Ärztegesellschaften entsprochen und Dr. med. Raphael Stolz ins Vizepräsidium dieser Versammlung gewählt.

Aktuell sieht die Aufgabenteilung für MPA- und MPKBelange wie folgt aus:

- Delegierte der kantonalen Ärztegesellschaften für MPA-Fragen: Präsidentin Dr. A. Ahmed, Vizepräsident Dr. Raphael Stolz

- Schweizerische Kommission für Berufsentwicklung und Qualität (Kommission B\&Q): Präsident Dr. J. Orellano

Im Berichtsjahr ist jede kantonale Ärztegesellschaft wieder in der Delegiertenversammlung vertreten.

- Aufgabenkommission für das Qualifikationsverfahren (QV) MPA: Präsident Dr. G. Printzen

- OdA Berufsbildung MPA (odamed): Präsidentin Frau M. Schenk/SVA; Vizepräsident Dr. G. Printzen/FMH

- Aufsichtskommission überbetriebliche Kurse (ÜK), Präsident Dr. Carlos Quinto, Vizepräsident Dr. José Orellano

- Die Anliegen der verschiedenen Gremien an den Zentralvorstand der FMH werden durch Dr. Carlos Quinto, Mitglied des Zentralvorstands der FMH sowie Departementsverantwortlicher Public Health und Gesundheitsberufe, vertreten.
- MPA-Kompetenzzentrum bei der FMH: Frau Annik Rüedi und Frau Chiwith Baumberger

Neu trifft sich das MPA-Kompetenzzentrum, das aus den oben aufgeführten Gremienverantwortlichen der FMH besteht, in regelmässigen Abständen, das heisst circa einmal monatlich, zu einer Onlinesitzung, um aktuelle Projekte und Anliegen im MPA/MPK-Bereich zu besprechen. So sind steter Austausch und Information aller Beteiligten optimal gewährleistet. Bei diesen Besprechungen werden wir unterstützt von Frau Barbara Weil, Leiterin der Abteilung Public Health der FMH, und Herrn lic. iur. Nils Graf vom Rechtsdienst der FMH.

Es folgen einige Informationen aus der Berichtsperiode im Überblick:

Die Delegiertenversammlung der kantonalen Delegierten für MPA-Fragen hat das Organisationsreglement in Kraft gesetzt.

Erfreulicherweise konnten in diesem Jahr auch alle Vakanzen gefüllt werden, so dass nun jede kantonale Ärztegesellschaft wieder in unserer Delegiertenversammlung vertreten ist.

Das MPA-Kompetenzzentrum soll in Zukunft vermehrt in Public-Health-Themen eingebunden werden. Ein weiteres Projekt war die Erstellung einer neuen Website für MPA-Belange, inklusive neuen Logos. Seit dem 1. August ist die neue Website aufgeschaltet: mpaschweiz.fmh.ch, Feedback ist willkommen.

In Zusammenarbeit mit dem SVA und ARAM wird der Musterarbeitsvertrag für MPA/MPK erneuert. Ferner hat die FMH klar Position bezogen, dass das praktische QV 2021 in allen Kantonen wie vor der Pandemie durchgeführt werden soll. 


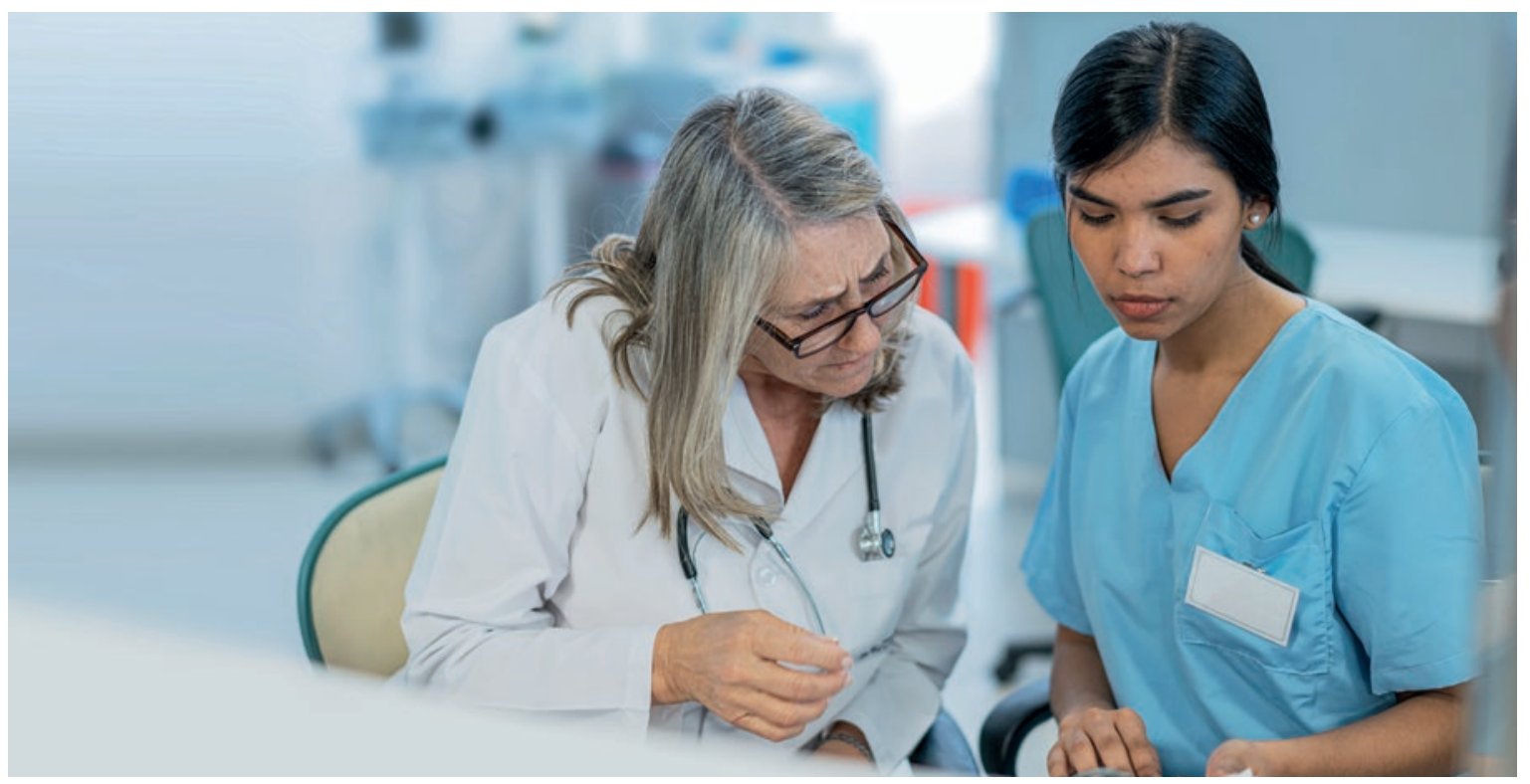

\section{Berichte aus den Kommissionen}

\section{Schweizerische Kommission für Berufsentwicklung und Qualität (Dr. José Orellano)}

Auch unter den Einschränkungen der Pandemie ist es zentral, dass Absprachen, Koordination und gemeinsame Entscheide im Team getroffen werden. Für die Kommissionsmitglieder war es nicht möglich, sich physisch zu treffen, so dass die Sitzungen im November 2020 und April 2021 erneut via Videokonferenz stattfinden mussten. Obwohl die Onlinemeetings mittlerweile sehr gut funktionieren und auch das Einloggen bei allen problemlos klappt, wünschen sich die Kommissionsmitglieder wieder eine Sitzung vor Ort.

Die B\&Q-Kommission und die Verbände hatten sich für die Durchführung des QV 2021 in einem normalen Rahmen ausgesprochen. Somit konnten die praktischen Abschlussprüfungen unter Einhaltung der vom BAG vorgegebenen Hygiene- und Verhaltensregeln durchgeführt werden. Bei den schriftlichen Prüfungen verhielt es sich wie im Jahr 2020, nämlich dass der berufskundliche Teil nicht geprüft, sondern der Gesamtschnitt aller Noten genommen wurde. So konnte die letzte Prüfung nach der alten Bildungsverordnung schweizweit unter gleichen Bedingungen abgeschlossen werden.

Die neue Bildungsverordnung wurde bisher gut umgesetzt. Die Vorbereitungen für das erste QV im Jahr 2022 gehen gut voran. Bei den Abschlussprüfungen mussten einige Anpassungen vorgenommen werden, damit sich die Handlungskompetenzen der Absolvierenden für die Prüfungen aussagekräftig genug bewerten lassen. Gemäss Aufgabenkommission werden die Chefexpertenkurse Anfang November 2021 gemeinsam mit der Eidgenössischen Hochschule für Berufsbildung (EHB) durchgeführt und im Anschluss daran die Schulungen der Prüfungsexpertinnen und -experten.

Die Ausführungsbestimmungen für das QV mit Validierung von Bildungsleistungen werden nach Aussprache mit den Gremien der Schweizerische Berufsbildungsämter-Konferenz (SBBK) und des Staatssekretariats für Bildung, Forschung und Innovation (SBFI) noch weiter an die jetzigen Standards angepasst, so dass diese ab 1.1.2022 in Kraft treten können.

Nach Beginn der Nullserie für das kommende QV 2022 wurde festgestellt, dass die Ausführungsbestimmungen «QV mit Abschlussprüfung» angepasst werden mussten. Dies aus dem Grund, da die medizinischen Grundlagen sonst zu kurz gekommen wären und zu wenig umfassend hätten geprüft werden können.

Ein steter Diskussionspunkt in der Kommission ist die Situation der Berufsbezeichnung «MPA». In der gesamten Schweiz ist es verbreitet, dass Berufsfachpersonen aus anderen Gesundheitsberufen in Praxen arbeiten, jedoch in der Funktion von MPA angestellt werden. Es kommt auch immer wieder zu Diskussionen bezüglich des Angebots verschiedenster Schulen mit Weiterbildungsangeboten im Bereich Grundbildung MPA. Ein offener Markt bietet diesen Institutionen viel Spielraum, so dass sie anbieten, was gemäss ihrer Einschätzung an Bedarf vorliegt. Festgestellt wurde, dass zwar die Inhalte beziehungsweise Handlungskompetenzen aus der Bildungsverordnung in den angebotenen Kursen vermittelt werden; jedoch entspricht der Lerninhalt nicht der Anzahl Lektionen, welche in der Bildungsverordnung vorgegeben werden. 
Häufig kommen Anliegen von Institutionen an die B\&Q-Kommission wie zum Beispiel, dass die erweiterte konventionelle Aufnahmetechnik (EKA) in die Grundbildung aufgenommen werden sollte. Dazu sagen allerdings SBFI und die Abteilung Strahlenschutz des BAG, dass es gemäss Strahlenschutz-Ausbildungsverordnung nicht zulässig ist, diese in der Grundbildung zu vermitteln.
Der SVA hat die Lerndokumentation überarbeitet. Sie ist nun online auf www.sva.ch erhältlich und wird gratis zur Verfügung gestellt, wofür sich die FMH bedankt. Vielen Dank ebenso an die Verbände ARAM und SVA für die gute Zusammenarbeit.

\section{Weiterbildung MPA - Bericht aus der OdA Berufsbildung MPA (Dr. Gert Printzen)}

Wie auch in den vergangenen Jahren wurden die obligaten Prüfungen für MPK in den Monaten November und Juni durchgeführt. Das Interesse daran ist nach wie vor ungebrochen. Am 24. November 2020 erfolgte die 11. Berufsprüfung zur medizinischen Praxiskoordinatorin mit 52 Kandidatinnen klinischer und 107 Kandidatinnen praxisleitender Fachrichtung. Darunter befanden sich 29 Repetentinnen und wiederum eine Kandidatin, welche die zweite Fachrichtung absolviert hat. 113 dieser total 159 Kandidatinnen schlossen die Prüfung erfolgreich ab - das entspricht zwar einer Erfolgsquote von $71 \%$, gleichwohl haben immerhin 29\% (46 Prüflinge) nicht bestanden, davon 13 Repetentinnen.

Am 29. Juni 2021 traten zur 12. Berufsprüfung zur medizinischen Praxiskoordinatorin 117 Teilnehmerinnen an, 25 davon Repetentinnen. 76 Teilnehmerinnen haben die Prüfung erfolgreich absolviert. Leider haben 41 Teilnehmerinnen, davon 11 Repetentinnen, die Prüfung nicht bestanden. Die 41 Kandidatinnen, die nicht bestanden hatten, sowie drei weitere Kandidatinnen wurden ein zweites Mal geprüft. Die Kommission hat schliesslich das Nichtbestehen der 41 Kandidatinnen bestätigt.

Der Termin für die kommende 13. Berufsprüfung 2021 ist der 23. November, und die Termine für die Berufsprüfungen 2022 sind für den 28. Juni und den 29. No- vember 2022 anberaumt. Für den dreizehnten Berufsprüfungstermin am 23. November 2021 sind bereits eindrucksvolle 145 Kandidatinnen (davon 63 klinisch und 81 praxisleitend) gelistet; hierunter befinden sich eine französischsprechende und zwei italienischsprechende Prüfungsteilnehmerinnen.

Die Finanzen der odamed stellen sich ausgeglichen dar, und die MPK-Betriebsphase präsentiert sich weiterhin erfolgreich. Die Abläufe werden wie bis anhin im Rahmen des Möglichen kontinuierlich professionalisiert; dazu gehören auch die ständige Optimierung der Website www.odamed.ch, die Weiterführung des Ausbaus auf drei Sprachen sowie die Akkreditierung weiterer Bildungsanbietender und Module.

Der Bedarf an Expertinnen und Experten in allen drei Landessprachen besteht wie bisher, und Interessierte werden gebeten, sich bei der Geschäftsstelle zu melden. Diese zukünftigen Expertinnen und Experten werden natürlich adäquat in ihre spezifische Aufgabe eingeführt und dafür geschult.

Ferner wird die Prüfungsordnung im laufenden sowie auch voraussichtlich im kommenden Jahr der Überprüfung beziehungsweise einer Revision unterzogen. Hieran arbeitet die dazu von der Qualitätssicherungskommission (QS) eingesetzte Arbeitsgruppe. Dem Ergebnis darf gespannt entgegengesehen werden!

\section{Aufgabenkommission für das Qualifikationsverfahren (QV) MPA (Dr. Gert Printzen)}

Was für ein Jahr liegt hinter uns! Wenn auch die Aufgabenkommission Qualifikationsverfahren QV MPA wie bis anhin mit grossem Engagement und voller Elan die Prüfungsunterlagen für das QV 2021 zusammenstellte, so haben uns das Coronavirus, die Beschränkungen der Pandemie und auch die Fachgruppen der Aufgabenkommission physisch und psychisch belastet. Die Erarbeitung unserer gesamtschweizerischen, einheitlichen Prüfung auf «digitalem Wege» war eine zusätzliche Herausforderung.
Schon das QV 2020 ging bereits unter dem Aspekt "Coronavirus(COVID-19)-Pandemie» in unsere Annalen ein. «Lessons Learned» daraus waren Bestrebungen, ein digitales $Q V$ zu generieren. Dies vor allem auch mit Blick auf die Umsetzung der neuen handlungskompetenzorientierten Bildungsverordnung vom Januar 2019. Das nun diesjährig aufgegleiste neue und innovative Prüfungskonzept erlaubt zudem den Kantonen sowohl die digitale als auch die bewährte Durchführung vor Ort des bzw. des kommenden QV MPA. 
Wenn die Umsetzung des oben angesprochenen Konzeptes gleichwohl für unser QV 2021 noch nicht reichte, so haben wir zumindest bis Juli dieses Jahres, also in dieser Berichtsperiode, eine funktionierende schriftliche Nullserie für das eQV 2022 fertigstellen und erfolgreiche Probeläufe mit ausgezeichneten Ergebnissen in einigen Kantonen durchführen können.

Für unser QV 2021 konnten unter Einhaltung der Hygieneregeln und anderer Vorgaben 1019 Prüflinge antreten. Die Gesamtzahl der Geprüften ist somit im Vergleich zum Jahr 2019 gesunken (1019 vs. 1057). Im zur Berichterstellung vorliegenden gesamtschweizerischen Überblick der Noten zeigt sich, dass die Zahl der Ungenügenden im Vergleich zum Jahr 2019 jedoch gesunken ist (75 bzw. 7,4\% vs. 99 bzw. 9,4\%) - obwohl die aktuelle Prüfung als nicht sonderlich einfach eingestuft worden war. Einmal mehr als mühsam gestaltete sich die Zusammenstellung der Prüfungsresultate zu einer Gesamtschau, da die Daten der Kantone zu extrem unterschiedlichen Zeitpunkten zur Verfügung gestellt wurden. Auch dies dürfte durch das kommende eQV erheblich erleichtert werden.

In dieser vergangenen Prüfungsperiode war der Aufwand für das QV wie in den vorhergehenden Jahren beträchtlich und konnte nur dank des engagierten Einsatzes aller Beteiligten bewältigt werden. Mein herzlicher Dank gilt einmal mehr allen Mitwirkenden für ihr bemerkenswertes und konstruktives Engagement!

\section{MPA-Ausbildung}

Im Jahr 2021 wurden 1019 MPA-Kandidatinnen geprüft. Von diesen 1019 Kandidatinnen haben leider 75 Kandidatinnen (7,4\%) die Prüfung nicht bestanden.

\section{Schlichtungsstelle}

Auch in dieser Berichtsperiode sind weder neue Vermittlungsgesuche beim Schlichter für die Deutschschweiz, Rechtsanwalt und Notar lic. iur. A. Kummer, eingegangen, noch sind Fälle hängig.

\section{Zum Schluss}

Die diesjährige MPA-Delegiertenversammlung wollten wir in der Form eines World Cafés interaktiver gestalten und aktuelle Themen im MPA-Bereich in einem lebendigeren Rahmen diskutieren. Wir haben deshalb die Delegierten gebeten, uns vorgängig Rückmeldungen zu folgenden Fragen zu geben:

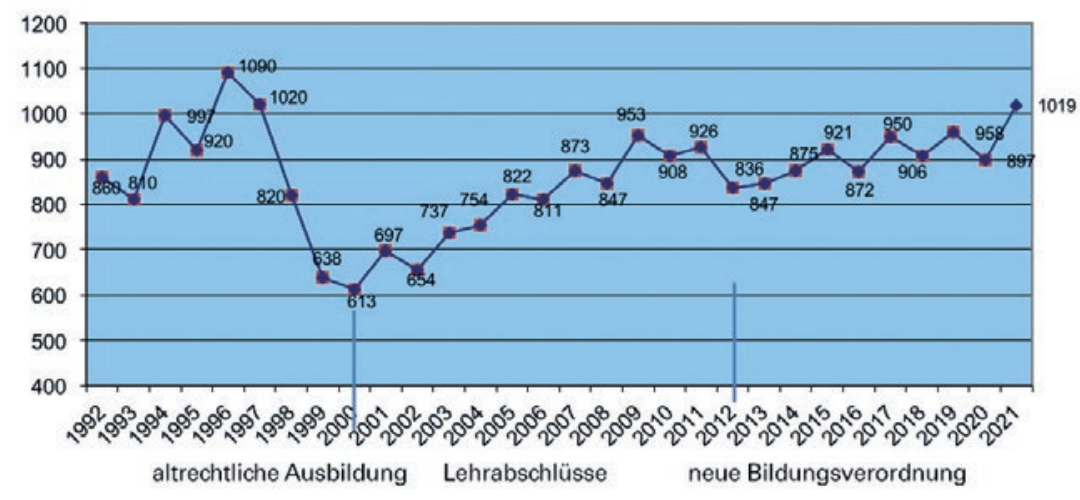

Im Jahr 2021 wurden 1019 MPA-Kandidatinnen geprüft.
- Welche Erwartungen haben Sie grundsätzlich an die Delegiertenversammlung?

- Haben Sie spezifische Wünsche/Themen, welche Sie an der Delegiertenversammlung vertieft diskutieren möchten?

- Gibt es Themen seitens Ihrer kantonalen Ärztegesellschaft, welche Sie an der Delegiertenversammlung einbringen möchten?

- Haben Sie Ideen zur Gestaltung und Finanzierung des Berufs MPA/MPK, um ihn weiterhin attraktiv halten zu können?

- Wie bewerben Sie den Beruf der MPA in ihrem Kanton?

Frau Barbara Weil übernahm die Leitung des World Cafés. Über die an diesem Anlass gewonnenen Erkenntnisse und Ideen, die Feedbacks und Anregungen werden wir Sie gerne zu einem späteren Zeitpunkt informieren.

Ich danke allen, die sich tatkräftig für die Belange der MPA und MPK einsetzen. In diesen Dank schliesse ich neben den Kommissionsverantwortlichen und den Delegierten insbesondere auch alle ein, die Ausbildungsstellen für MPA anbieten, sowie die Berufsbildnerinnen und -bildner, die Lehrpersonen an den Berufsschulen und die MPA-Verbände. Spezieller Dank gebührt Frau Annik Rüedi und Frau Chiwith Baumberger, welche das MPA-Kompetenzzentrum sehr motiviert und mit grossem Einsatz führen.

Bildnachweis

iStock.com/Authentic Images 